\title{
EMS Response Time for Patients Critically-Injured from Automobile Accidents Using Regression Analysis
}

\author{
Sneha R. Vanga, Phillip M. Ligrani, Mehrnaz Doustmohammadi, Michael Anderson \\ Department of Mechanical and Aerospace Engineering, Department of Civil \& Environmental Engineering, The University of \\ Alabama in Huntsville, Huntsville, AL, USA \\ Email:pml0006@uah.edu
}

How to cite this paper: Vanga, S. R., Ligrani, P. M., Doustmohammadi, M., \& Anderson, M. (2021). EMS Response Time for Patients Critically-Injured from Automobile Accidents Using Regression Analysis. Current Urban Studies, 9, 581-596. https://doi.org/10.4236/cus.2021.93035

Received: August 8, 2021

Accepted: September 19, 2021

Published: September 22, 2021

Copyright $\odot 2021$ by author(s) and Scientific Research Publishing Inc. This work is licensed under the Creative Commons Attribution International License (CC BY 4.0).

http://creativecommons.org/licenses/by/4.0/

\begin{abstract}
Background: The ability to quickly and effectively receive medical treatment in the event of an automobile collision is one of the most important aspects in emergency medical services (EMS). Emergency medical service providers are the first to respond and manage cases related to trauma, emergency surgery, and critically injured patients. Response time for emergency medical services vehicles is especially important for areas, where travel distances are often much larger, compared to more urban areas. The importance of the present data and analysis procedures are their applicability to multiple environments, including urban settings. Methods: The present study is focused on optimization of analysis tools, and understanding the influences of different trafficrelated variables, related to hospital EMS transport times for Pickens County, a county in west Alabama. Optimization of associated analysis tools is important for optimal trauma patient survivability, and as such, is directly relevant to the management of care for severely injured surgical patients. Of particular interest are the effects of variables, such as travel time, time of the day, day of the week, weather, lighting conditions, and crash severity, on the EMS response time (ERT), which are analyzed using two types of advanced regression analysis: geographically weighted regression (GWR) and global regression analysis (GRA). Results: For GWR analysis, the accuracy of the approach is improved by employing an adaptive bi-square kernel weighting function. The GWR approach is also unique because geographic location variations are quantified for local independent variables, as their effects are included. Magnitudes of variable coefficients, and variable t-statistic values provide information on the relative influences and impacts of different variables, and different variable combinations, as they are considered in pairs, triplets, and different combinations. Conclusion: The resulting effects and alterations to optimal
\end{abstract}


EMS response time are provided for a wide range of travel conditions and travel time periods.

\section{Keywords}

Automobile Collision, Emergency Medical Services (EMS), EMS Response Time, Geographically Weighted Regression (GWR), Global Regression Analysis (GRA)

\section{Introduction}

Recent reports from the World Health Organization (WHO) indicate that road traffic injuries (RTIs) account for about 1.3 million deaths worldwide annually (World Health Organization, 2013; World Health Organization, 2015). Peden, Scurfield, Sleet, Mohan, Hyder and Jarawan (2004) indicate that the RTI rate of mortality is continually increasing around the world, especially in low- and middle-income countries (LMICs). Additional analysis of road traffic accidents within LMICs by Eftekhari, Khorasani-Zavareh and Nasiriani (2018), and Gopalakrishnan (2012) additionally shows that most RTI deaths occur prior to hospital arrival, either at the at the crash scene, or during patient transport. According to Eftekhari, Khorasani-Zavareh and Nasiriani (2018), and Bakke, Steinvik, Eidissen, Gilbert and Wisborg (2015), 86 percent of trauma-related deaths occur in the pre-hospital phase; and about 39 percent of these deaths are preventable.

In spite of important improvements to road infrastructure, vehicle design, and traffic safety legislation within the United States in recent years, motor vehicle crashes (MVC) also continue to be a leading cause of death and injury (National Highway Traffic Safety Administration, 2017). Because emergency medical services (EMS) provide the critical link between injury and definitive critical care (Insurance Institute for Highway Safety Highway Loss Data Institute, 2015), the time between the occurrence of a MVC and delivery of a patient to this care is a vital factor in regard to the potential and probability of MVC mortality (National Academies of Sciences, Engineering, and Medicine, 2016). According to Brown, Sajankila and Claridge (2017), early arrival of EMS at a crash scene generally leads to stabilization of occupants with life threatening injuries, timely triage and transport to a hospital. Reducing EMS delays associated with such activities will lead to lower probability of loss of life (Kumar, Abudayyeh, Fredericks, Kuk, Valente, \& Butt, 2017; Byrne, Mann, Dai, Mason, Karanicolas, Rizoli, \& Nathens, 2019), which has direct consequences in regard to the quality of life for individuals at risk. Byrne, Mann, Dai, Mason, Karanicolas, Rizoli and Nathens (2019) provide results from a population-based analysis from 2268 US counties, which demonstrates a definitive connection between longer emergency medical services (EMS) response times and higher rates of motor vehicle crash (MVC) mortality. Of particular importance are results for both rural/wilderness and urban/ 
suburban settings, where a significant proportion of crash fatalities $(9.9 \%$ and $14.1 \%$, respectively) are associated with prolonged response times. Other investigators also demonstrate that the time taken for EMS to reach a crash site is strongly influenced by the location (rural, suburban, or urban) of the crash site (Gonzalez, Cummings, Phelan, Mulekar, \& Rodning, 2009; NCHRP, 2013). Additional early investigations of the consequences, effects, relationships, and associated factors in regard to MVC's, ERT, and patient mortality are described by Evanco (1999), Lambert \& Meyer (2006), Gonzalez, Cummings, Phelan, Mulekar, \& Rodning (2009), Trowbridge, Gurka, \& O'Connor (2009), and Griffin \& McGwin (2013). Within these investigations, response time or travel time is the interval between the initial reporting of a crash and the arrival of EMS personnel at the crash site (Pons, Haukoos, Bludworth, Cribley, Pons, \& Markovchick, 2005; Lambert \& Meyer, 2006; Gonzalez, Cummings, Phelan, Mulekar, \& Rodning, 2009).

Eftekhari, Tafti, Nasiriani, Hajimaghsoudi, Fallahzadeh and Khorasani-Zavareh (2019) identify and discuss issues related to preventable deaths due to RTIs in the prehospital phase of events resulting from a MVC. Data are obtained from 24 RTI prevention experts, which show that poor management of time is one of the six major challenges related to preventable deaths in RTIs in the prehospital phase. Another recent study by Ma, Zhang, Yan, Wang, Song and Xiong (2019) indicate that ERT, in addition to age, gender, seating position, and manner of collision, are all statistically significant in regard to the possibility of a fatality. He, Qina, Renger and Souvannasacd (2019) use spatial regression methods to show that establishing EMS performance measures is critical for the improvement of a rural community's access to Emergency Medical Services (EMS). Results from this analysis show that low service coverage measure means that additional strategic establishment or relocation of service stations are needed.

The present study is focused on optimization of analysis tools, and understanding the influences of different traffic-related variables, related to hospital EMS transport times for Pickens County, a county in west Alabama. This particular county is selected for analysis because travel distances are often much larger, and EMS Response Times are often longer, compared to more urban areas. This particular county is also unique because it includes only one EMS dispatch center. The importance of the present data and analysis procedures are their applicability to multiple environments, including urban settings.

Of particular interest is determination of the optimal and most physically-realistic analysis approach, which considers the effects of six independent variables (travel time, time of the day, day of the week, weather, lighting conditions, crash severity) on one dependent variable, ERT, the EMS response time. The study is undertaken using two types of advanced regression analysis: geographically weighted regression (GWR) and global regression analysis (GRA). The present investigation thus demonstrates the application and use of these analytic tools for determination of the dependence and impact of ERT in regard to different com- 
binations of independent variables. The GWR analysis approach is unique because geographic location variations are included for local independent variables as they are incorporated within the analysis, which is accomplished using an adaptive bi-square kernel weighting function. This particular kernel function incorporates a distance decay function, which allocates more weight to properties closer to a regression point, than to properties farther away. Understanding the effect of different independent variables on ERT is important, because shortening the ERT minimizes the complications that can ensue related to minor injuries, and major injuries, and reduces the possibility of fatalities. Optimization of associated analysis tools is thus vital for optimal trauma patient survivability, and as such, is directly relevant to the management of care for severely injured surgical patients.

\section{Methods}

\subsection{Test Environment Data}

Pickens County is a county located on the west central border of the U.S. state of Alabama. Pickens County has a medical center located at 241 Robert K Wilson Dr., Carrollton, AL 35447. This is the only EMS dispatch location in the county. The longitude and latitude coordinates for the Emergency Medical Services (EMS) dispatch center in Pickens County are -88.086 and 33.276, respectively.

Crash records for Pickens County, Alabama were extracted from the Critical Analysis Reporting Environment (CARE) database for the dates between January 2016 and December 2019, resulting in a total of 214 crash cases. The crashes used in the study were only those for which EMS was dispatched. The variables considered are EMS response time (ERT), actual travel time, crash severity, day of the week, time of the day, lighting condition, and weather. Travel time is calculated between the Pickens County hospital location and each of the crash sites using Google Maps with travel time recorded in minutes for the fastest route. Data are analyzed using two methods-Global Regression Analysis (GRA) and Geographically Weighted Regression (GWR) analysis. GWR4 software is used to analyze the data using both the analysis methods and each variable from the data is divided into different categories by code numbers. Figure 1 shows a map of Pickens County, with the hospital (EMS) location, and including four examples of crash site locations.

\subsection{Regression Analysis Approach-Geographically Weighted Regression (GWR)}

Two types of regression analysis are considered: GWR or Geographically Weighted Regression, and GRA or Global Regression Analysis. To implement these analysis approaches, GWR4 software is employed. GWR4 is statistical software developed especially for GWR modeling (Nakaya, 2014). Within this software package, the semi-parametric Gaussian GWR model is described using the following equation 


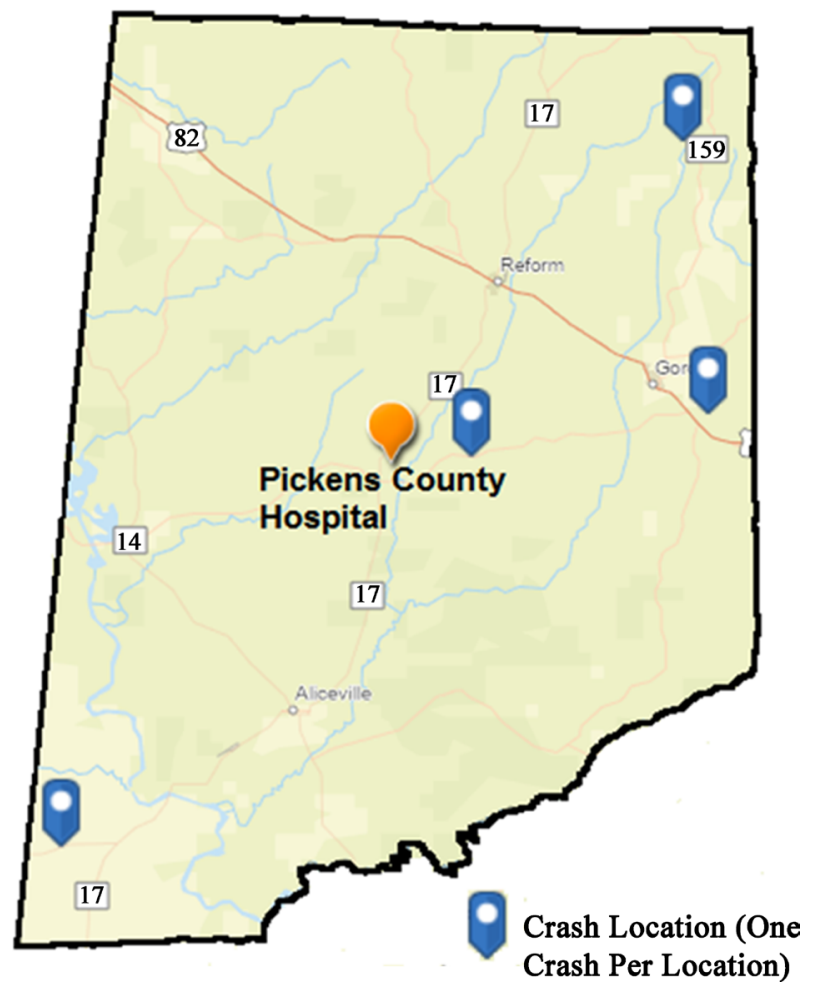

Figure 1. Pickens County map with hospital (EMS) location and four examples of crash sites, with one crash per site.

$$
y_{i}=\sum_{k} \beta_{k}\left(u_{i}, v_{i}\right) x_{k, i}+\sum_{l} \gamma_{l} z_{l, i}+\varepsilon_{i}
$$

where $y_{i}, x_{k, i}$ and $\varepsilon_{i}$ are the dependent variable, $k$ th independent variable, and the Gaussian error at the location $i$, respectively. Parameter variables $\left(u_{i}, v_{i}\right)$ are the latitude and longitude coordinates of the $i$ th location, and coefficients $\beta_{k}\left(u_{i}, v_{i}\right)$ are varying with location. Hence, $x_{k, i}$ are considered as local variables. $z_{l, i}$ is the $i$ th independent variable with a fixed coefficient $\gamma_{l}$ and does not vary with location. Variable $z_{l, i}$ is thus a global parameter. Thus, the model uses both geographically local terms and global terms.

For GWR analysis, the coefficient $(\beta)$ for the local variables is not constant like the global variables. The coefficient $(\beta)$ value varies based on the geographical location. Hence, instead of an estimate for the coefficient, for the GWR results, the mean, standard deviation, minimum, and maximum values of the coefficient are provided for the local variables. Whereas for the global variables, the coefficient estimate, standard error (SE) and the t-statistic values are provided. The standard error (SE) is an estimate of the standard deviation of the coefficient, the amount it varies across cases. It can be thought of as a measure of the precision with which the regression coefficient is measured. The $t$ statistic is the coefficient estimate divided by its standard error. The $t$ statistic value indicates how strongly each independent variable is associated with the dependent variable.

A Gaussian model with an adaptive bi-square kernel weighing function is used with the geographically weighted regression (GWR) analysis. In GWR modeling, 
parameters for a location are more strongly affected by the observations occurring close by, as opposed to observations made from farther away. The associated influence factor is called the weighting function, wij. Observation is a case of crash data at one particular location. The weighting function value for each case (crash data) indicates the influence of this case on the regression estimate of a different crash case. The weighting function value of cases closer in location to a particular crash case is higher, relative to the weighting function value of cases which are farther away. An adaptive bi-square kernel weighting function is employed within the present study to implement these variations, as expressed by the equation given by

$$
w_{i j}= \begin{cases}\left(1-\left(\frac{d_{i j}}{h_{i(k)}}\right)^{2}\right)^{2} & \text { if } d_{i j}<h_{i(k)} \\ 0 & \text { otherwise }\end{cases}
$$

With this approach, the bi-square kernel assigns a weight of zero to observations outside of the bandwidth, nullifying their impact on the regression estimate. Within Equation (2), $i$ is the regression point index. $j$ is the locational index. $w_{i j}$ is the weight value of observation at location $j$ for estimating the coefficient at location $i . d_{i j}$ is the Euclidean distance between $i$ and $j . h$ is a fixed bandwidth size defined by a distance metric measure, and $h_{i(k)}$ is an adaptive bandwidth size defined as the $k$ th nearest neighbor distance.

With the GWR approach, the adaptive kernel is employed to optimize the size of the bandwidth hi through use of the number of neighboring observations. This is accomplished as the bi-square kernel function incorporates a distance decay function, which allocates more weight to properties closer to a regression point, than to properties farther away.

\subsection{Regression Analysis Approach-Global Regression Analysis (GRA)}

Global regression analysis does not account for geographical variability. For global regression analysis, the model is expressed using an equation of the form

$$
y_{i}=\sum_{k} \beta_{k} x_{k, i}+\varepsilon_{i}
$$

where $y_{i}, x_{k, i}$ and $\varepsilon_{i}$ are dependent variable, $k$ th independent variable, and the Gaussian error, respectively. Here, a different $\beta$ coefficient is employed for each variable.

\section{Variable Selection and Analytic Results}

\subsection{Variable Selection and Resulting Data Trends}

EMS response time (ERT) is the dependent variable. Independent variables are day of the week, time of day, weather, crash severity, lighting conditions, and travel time. For the GWR4 software to process the data, different code numbers are used to represent different range magnitudes of the variables. Note that, by 
changing the code numbers for these variables, the intercept changes but the $\beta$ coefficient remains the same. Also note that reversing the number order changes the sign of coefficient for a particular variable.

Figure 2(a) shows the EMS response time (ERT) variation with travel time. The line in the plot indicates ERT is equal to the travel time. ERT is longer than travel time for most cases. This is because EMS requires additional time for the initial communication, unit dispatch time, and scene time. In few cases, ERT is

(a)

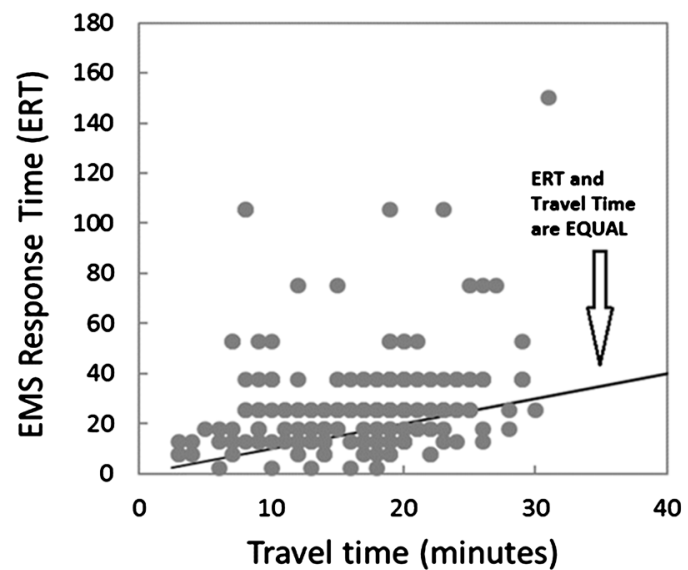

(b)
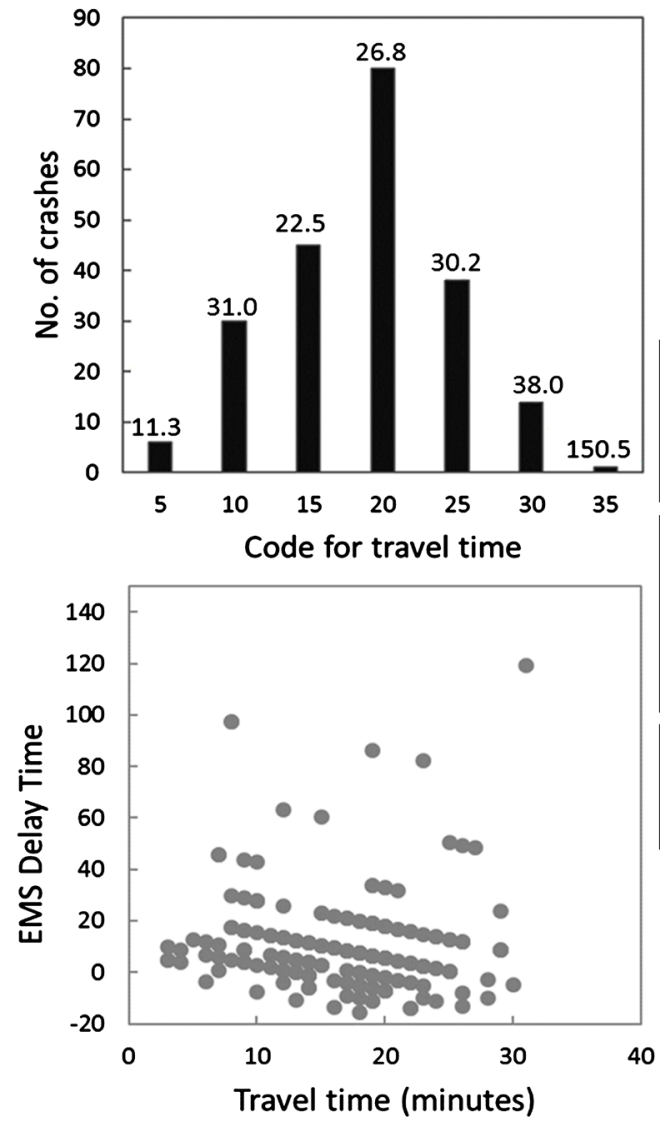

MS Response Time (ERT):

$2.5-0$ to 5 minutes

$8-6$ to 10 minutes

13 - 11 to 15 minutes

18 - 16 to 20 minutes

25.5 - 21 to 30 minutes

38 - 31 to 45 minutes

75.5 - 61 to 90 minutes

105.5 - 91 to 120 minutes

$150.5-121$ to 180 minutes . 
shorter than travel time. This is because the EMS unit travels faster than normal driving limits, and sometimes, the EMS unit is available closer to the crash location than the dispatch center.

Figure 2(b) histogram shows the variation of the number of crashes with code for the variable, travel time. Here, the travel time interval associated with the largest number of crashes is from 16 to 20 minutes. Note that the average ERT is given above the histogram bar for each code value within Figure 2(b). Average ERT values within this figure generally show the expected trend of an increase with travel time, with one exception. The average ERT value is higher than expected for the 6 to 10 minute travel time interval, because of anomalous events, with larger delay times, associated with more serious injuries and longer than typical scene times.

Figure 2(c) shows the variation of EMS delay time with travel time. Here, EMS delay time is the difference between EMS response time and travel time. Negative delay time values thus indicate that ERT is smaller than travel time. The trend of data within Figure 2(c) shows that delay time generally decreases as travel time increases. This means that, as travel distances become larger, and travel times are larger, differences between EMS response time and travel time are generally less.

\subsection{Global and Local Variables Using Diff-Criterion for GWR Results with Adaptive Bi-Square Kernel Weighting Function}

To determine if a variable is global or local, a geographical variability test is performed. Geographical variability for each coefficient is tested by model comparison. For testing the geographical variability of the $k$ th varying coefficient, a model comparison is carried out between the original GWR model ( $k$ th variable as local) and a switched GWR model in which only the $k$ th coefficient is fixed (as global variable) while other coefficients are kept as they are in the original GWR model. If the original local GWR ( $k$ th variable as local) is better than the switched GWR model ( $k$ th variable is fixed) by a model comparison criterion (such as a corrected Akaike Information Criterion, AICc, as described by He, Qina, Renger and Souvannasacd (2019), the $k$ th coefficient is certainly varying over space. The test routine repeats this comparison for each geographically varying coefficient. Difference of criterion (diff-Criterion) is the comparison of criterion value between original GWR model ( $k$ th variable as local) and switched GWR model ( $k$ th variable as global). Negative value of the difference indicates that the original GWR model is better than the switched GWR model, which indicates that the $k$ th variable is local or spatially-varying. Positive value of diff-Criterion suggests no spatial variability and indicates that the $k$ th variable is global (fixed). Diff-Criterion values are high and negative except for travel time which is positive and small. Local variables vary geographically. Global variables are fixed. From Diff-Criterion results, independent variables, day of the week, time of the day, weather, crash severity, and lighting conditions are local terms, whereas 
travel time is a global term.

\subsection{Geographically Weighted Regression (GWR) Results, With Adaptive Bi-square Kernel Weighting Function}

As mentioned, all independent variables, except travel time, are local terms. Travel time is the only global term. For GWR analysis, coefficient $\beta$, included within Equation (1), is associated with each local variable. Coefficient $\beta$ is not constant like the global variables, but varies based on the geographical location. Hence, instead of an estimate for the $\beta$ coefficient, for the GWR results, the mean, standard deviation, minimum, and maximum values of the coefficient are provided for the local variables, which are day of the week, time of the day, weather, crash severity, lighting conditions. In contrast, for the global variable travel time, provided are estimated value, standard error (SE) value, and t statistic value. For the analysis results which follow, the effect of the variable travel time is considered on an individual basis, as well as with combinations of other variables, in regard to the effects of independent variables on the dependent variable, EMS response time or ERT.

\subsubsection{Travel Time-Global Independent Variable}

Table 1 shows GWR results with adaptive bi-square kernel for ERT as dependent variable and travel time as only one independent variable. To provide a baseline case for comparison as effects of additional variables are included in the analysis, only travel time is considered as an independent variable. Based on the geographical variability test results, travel time is considered as a global variable. When only the effect of travel time on ERT is analyzed, travel time has a positive impact. This indicates that ERT increases with increase in the travel time. The coefficient estimate of travel time is 1.147 which indicates that ERT is longer than travel time. This means that the average ERT is $14.7 \%$ longer than actual travel time when no other independent variables are considered. The standard error (SE) which indicates the variation of estimate is 0.290 .

The greater the magnitude of t-statistic, the greater the evidence that there is a significant effect upon the dependent variable due to the independent variable. The t-statistic value is for travel time is 3.955 which indicates that travel time has a significant impact on the EMS response time (ERT).

\subsubsection{Travel Time-Global Independent Variable Consideration with Other Local Independent Variables}

Tables 2(a)-(d) show GWR results with adaptive bi-square kernel for ERT as

Table 1. Geographically weighted regression (GWR) analysis (with bi-square kernel weighting function) results for EMS response time (ERT) as the dependent variable, and one independent variable, travel time.

\begin{tabular}{ccccc} 
Type of variable & Independent variables & Coefficient estimate Standard Error & $\mathrm{t}$ (Estimate/SE) \\
\hline Global & Travel time & 1.147 & 0.290 & 3.955 \\
\hline
\end{tabular}


Table 2. (a) Geographically weighted regression (GWR) analysis (with bi-square kernel weighting function) results for EMS response time (ERT) as the dependent variable, and two independent variables, including travel time; (b) Geographically weighted regression (GWR) analysis (with bi-square kernel weighting function) results for EMS response time (ERT) as the dependent variable, and three independent variables, including travel time; (c) Geographically weighted regression (GWR) analysis (with bi-square kernel weighting function) results for EMS response time (ERT) as the dependent variable, and four independent variables, including travel time; (d) Geographically weighted regression (GWR) analysis (with bi-square kernel weighting function) results for EMS response time (ERT) as the dependent variable, and six independent variables, including travel time.

(a)

\begin{tabular}{|c|c|c|c|c|c|c|c|c|}
\hline $\begin{array}{l}\text { Type of } \\
\text { variable }\end{array}$ & $\begin{array}{c}\text { Independent } \\
\text { variables }\end{array}$ & $\begin{array}{l}\text { Coefficient } \\
\text { estimate }\end{array}$ & $\begin{array}{c}\text { Standard } \\
\text { Error }\end{array}$ & $\begin{array}{c}\mathrm{t} \\
\text { (Estimate/SE) }\end{array}$ & Mean & $\begin{array}{l}\text { Standard } \\
\text { Deviation }\end{array}$ & Minimum & Maximum \\
\hline Global & Travel time & 1.022 & 0.285 & 3.592 & & & & \\
\hline Local & Lighting condition & & & & 1.722 & 2.840 & -2.224 & 17.497 \\
\hline Global & Travel time & 1.159 & 0.245 & 4.730 & & & & \\
\hline Local & Weather & & & & 0.112 & 1.888 & -3.655 & 2.400 \\
\hline Global & Travel time & 1.230 & 0.261 & 4.720 & & & & \\
\hline Local & Day of the week & & & & 6.244 & 9.734 & -8.694 & 27.672 \\
\hline Global & Travel time & 1.066 & 0.296 & 3.606 & & & & \\
\hline Local & Time of the day & & & & 1.134 & 2.479 & -2.714 & 15.744 \\
\hline Global & Travel time & 1.233 & 0.329 & 3.751 & & & & \\
\hline Local & Crash Severity & & & & 0.703 & 5.706 & -44.274 & 18.124 \\
\hline
\end{tabular}

(b)

\begin{tabular}{|c|c|c|c|c|c|c|c|c|}
\hline $\begin{array}{l}\text { Type of } \\
\text { variable }\end{array}$ & $\begin{array}{l}\text { Independent } \\
\text { variables }\end{array}$ & $\begin{array}{l}\text { Coefficient } \\
\text { estimate }\end{array}$ & $\begin{array}{l}\text { Standard } \\
\text { Error }\end{array}$ & $\begin{array}{c}\mathrm{t} \\
\text { (Estimate/SE) }\end{array}$ & Mean & $\begin{array}{l}\text { Standard } \\
\text { Deviation }\end{array}$ & Minimum & Maximum \\
\hline Global & Travel time & 1.236 & 0.248 & 4.991 & & & & \\
\hline \multirow{2}{*}{ Local } & Weather & & & & -0.148 & 2.734 & -7.146 & 2.689 \\
\hline & Day of the week & & & & 7.459 & 10.097 & -4.774 & 29.708 \\
\hline Global & Travel time & 1.023 & 0.312 & 3.277 & & & & \\
\hline \multirow{2}{*}{ Local } & Time of the day & & & & 0.586 & 3.358 & -6.088 & 21.831 \\
\hline & Day of the week & & & & 5.529 & 11.339 & -36.046 & 35.340 \\
\hline Global & Travel time & 1.166 & 0.273 & 4.277 & & & & \\
\hline \multirow{2}{*}{ Local } & Weather & & & & 0.287 & 2.357 & -4.912 & 3.057 \\
\hline & Time of the day & & & & 1.293 & 1.962 & -1.982 & 10.353 \\
\hline
\end{tabular}

(c)

\begin{tabular}{|c|c|c|c|c|c|c|c|c|}
\hline $\begin{array}{l}\text { Type of } \\
\text { variable }\end{array}$ & $\begin{array}{c}\text { Independent } \\
\text { variables }\end{array}$ & $\begin{array}{c}\text { Coefficient } \\
\text { estimate }\end{array}$ & $\begin{array}{l}\text { Standard } \\
\text { Error }\end{array}$ & $\begin{array}{c}\mathrm{t} \\
\text { (Estimate/SE) }\end{array}$ & Mean & $\begin{array}{l}\text { Standard } \\
\text { Deviation }\end{array}$ & Minimum & Maximum \\
\hline \multirow[t]{2}{*}{ Global } & Travel time & 1.081 & 0.297 & 3.634 & & & & \\
\hline & Weather & & & & 0.205 & 3.328 & -10.477 & 4.505 \\
\hline \multirow[t]{2}{*}{ Local } & Time of the day & & & & 0.888 & 2.915 & -3.667 & 19.532 \\
\hline & Day of the week & & & & 6.080 & 11.934 & -31.468 & 41.220 \\
\hline
\end{tabular}


(d)

\begin{tabular}{|c|c|c|c|c|c|c|c|c|}
\hline $\begin{array}{l}\text { Type of } \\
\text { variable }\end{array}$ & $\begin{array}{l}\text { Independent } \\
\text { variables }\end{array}$ & $\begin{array}{l}\text { Coefficient } \\
\text { estimate }\end{array}$ & $\begin{array}{l}\text { Standard } \\
\text { Error }\end{array}$ & $\begin{array}{c}\mathrm{t} \\
\text { (Estimate/SE) }\end{array}$ & Mean & $\begin{array}{l}\text { Standard } \\
\text { Deviation }\end{array}$ & Minimum & Maximum \\
\hline Global & Travel time & 1.104 & 0.268 & 4.115 & & & & \\
\hline \multirow{5}{*}{ Local } & Time of the day & & & & -0.311 & 2.889 & -5.466 & 4.408 \\
\hline & Day of the week & & & & 6.683 & 10.115 & -6.382 & 33.278 \\
\hline & Weather & & & & -0.079 & 3.062 & -8.717 & 3.407 \\
\hline & Lighting Condition & & & & 1.237 & 2.820 & -3.271 & 8.798 \\
\hline & Crash severity & & & & 0.701 & 2.854 & -9.373 & 10.800 \\
\hline
\end{tabular}

dependent variable and travel time as one independent variable with additional independent variables added to the analysis. Overall, travel time has a positive impact on the ERT. It indicates that as the travel time increases, the ERT also increases. The coefficient of the variable travel time ranges between 1.022 and 1.236 as different independent variables are added to the analysis. The range of coefficients for travel time indicates that additional variables have an impact on ERT. Adding variables time of the day and lighting conditions to the analysis reduces the coefficient estimate for travel time. Whereas, adding variables weather, day of the week, and crash severity increases the coefficient estimate value.

The $t$-statistic value greater than 1 indicates the coefficient estimate is bigger than the standard error, which indicates that the independent variable has a significant effect upon the dependent variable. The t-statistic value for variable travel time varies between 3.277 and 4.991. This indicates that travel time has a significant impact on the EMS response time (ERT) variation. The t-statistic value for travel time increases by adding variables weather and day of the week to the analysis. But adding, variables time of the day, lighting condition, and crash severity to the analysis somewhat reduces the $t$-statistic value.

\subsection{Global Regression Analysis (GRA) Results}

Global regression analysis (GRA) does not account for spatial variability of the independent variables, which means that the model is without spatial variation relationships, and that model results are independent of spatial location. Global estimate values are spatial averages, and all independent variables are global. As such, global regression analysis is employed to provide spatially-independent results for comparison with GWR analysis results, where in spatial variations of independent variables are included.

Table 3 shows global regression results for ERT as dependent variable and travel time as one independent variable with five additional independent variables. Based on the coefficient estimates, when six independent variables are considered, data indicates that travel time has a higher impact on ERT with GWR analysis than global regression analysis. Day of the week also has a lower impact on ERT with global regression analysis. Weather, lighting condition, and crash severity 
have higher impact with global regression analysis than the GWR model. The impact of time of the day on ERT with global regression analysis is higher when compared to GWR with bi-square kernel. The t-statistic values show that variables travel time, day of the week, and lighting condition have statistically significant impact on ERT, whereas variables weather, time of day, and crash severity have lower significance.

\subsection{ANOVA Comparisons of GWR Results and GRA Results}

ANOVA, or analysis of variance, results provide comparisons between GRA, global regression analysis, and GWR, geographically weighted regression analysis. With this approach, Source, Sum of Squares (SS), Degrees of Freedom (DF), Mean Square (MS), and F-statistic value are determined. As such, ANOVA values indicate if adding geographical variability location data (by means of GWR) leads to an improvement in model performance. The sum of squares (SS) represents a measure of variation or deviation from the mean, and is calculated as a summation of the squares of the differences from the mean. Values of SS are calculated for GRA residuals, GWR residuals, and GWR improvement. The difference between the GRA residuals value and the GWR residuals value is the GWR improvement. GWR improvement is determined both for SS values and for DF values. The MS is calculated by dividing the Sum of Squares (SS) by the Degrees of Freedom (DF). The F-statistic is then the MS value of GWR improvement divided by the MS value of the GWR residuals.

Table 4 shows the ANOVA Table which indicates that SS is lower for GWR

Table 3. Global regression analysis (GRA) results for EMS response time (ERT) as the dependent variable, and six independent variables, including travel time.

\begin{tabular}{cccc}
\hline Independent variables & Coefficient estimate & Standard Error & $\mathrm{t}$ (Estimate/SE) \\
\hline Travel time & 0.696 & 0.221 & 3.148 \\
Time of the day & -0.552 & 1.700 & -0.325 \\
Day of the week & 4.845 & 2.890 & 1.677 \\
Weather & -0.134 & 1.292 & -0.104 \\
Lighting condition & 2.315 & 1.417 & 1.634 \\
Crash Severity & 1.246 & 1.566 & 0.795 \\
\hline
\end{tabular}

Table 4. ANOVA Table for six independent variables, analyzed using geographically weighted regression (GWR) with bi-square kernel weighting function, and global regression analysis (GRA).

\begin{tabular}{ccccc}
\hline Source & SS & DF & MS & F \\
\hline GRA Residuals & $72,547.941$ & 207.000 & & \\
GWR Improvement & $28,111.033$ & 46.317 & 606.922 & \\
GWR Residuals & $44,436.908$ & 160.683 & 276.551 & 2.195 \\
\hline
\end{tabular}


residual values than for GRA residual values. Because a lower SS value indicates a better fit model, results indicate that the GWR approach provides improved model analysis results. Table 4 also shows that the F-statistic is greater than 1, which indicates that the MS value of GWR improvement is higher than the MS value associated with GWR residuals. This result also provides evidence that an improved model fit is provided by the GWR approach.

\section{Summary and Conclusions}

The present investigation is undertaken to demonstrate the use of Geographically Weighted Regression (GWR) and Global Regression Analysis (GRA) for determination of the dependence of EMS Response Time (ERT) in regard to a variety of independent variables. EMS response time (ERT) variation data are provided for Pickens County, a county in west Alabama. The choice of this test environment is unique because only one EMS dispatch center is located within the county. The GWR analysis approach is unique because geographic location variations are included for local independent variables, as they are incorporated within the analysis, which is accomplished using an adaptive bi-square kernel weighting function. This particular kernel function incorporates a distance decay function, which allocates more weight to properties closer to a regression point, than to properties farther away. The present investigation thus demonstrates the application and use of these analytic tools for determination of the dependence and impact of ERT in regard to different combinations of independent variables. From geographical variability results, the local independent variables, which are considered, are day of the week, time of the day, weather, crash severity, and lighting conditions. The one global independent variable is travel time. As such, the present data and analysis procedures are important because of their applicability to multiple environments, including urban settings.

Of particular interest are the effects and influences of these six independent variables on one dependent variable, ERT, the EMS response time. From ANOVA table data, Sum of Squares (SS) values and Mean Square (MS) values are lower for GWR analysis with the adaptive bi-square kernel weighting function, relative to GRA determined values. The associated F-statistic is greater than 1, and equal to 2.195, which indicates that the Mean Square value of GWR improvement is higher than the Mean Square value associated with GWR residuals. Such results provide evidence that an improved model fit is provided by the GWR approach, which is considered to be the optimal and most physically-realistic analysis method. As such, improved model performance is provided by including geographic location variations for local independent variable effects, through the inclusion of an adaptive bi-square kernel weighting function.

Magnitudes of coefficients provide quantitative assessments of the impact and influence of different independent variables on the dependent variable, ERT. When only the effect of the travel time on ERT is analyzed, travel time has a positive impact. This indicates that ERT increases with increase in the travel 
time. The average ERT is 14.7 percent longer than actual travel time when no other independent variables are considered. As additional independent variables are added to the analysis, the coefficient of the variable travel time ranges between 1.022 and 1.236, which again indicates that ERT is longer than travel time for different combinations of variables. Adding variables time of the day and lighting conditions to the analysis reduces the coefficient estimate for travel time. Whereas adding variables weather, day of the week, and crash severity increases the coefficient estimate value. T-statistic values for variable travel time, ranging between 3.277 and 4.991, confirm that travel time has a significant impact on the EMS response time (ERT) variation. The t-statistic value for travel time increases by adding variables weather and day of the week to the analysis. However, adding variables, time of the day, lighting condition, and crash severity, to the analysis somewhat reduces associated t-statistic values. Note that the qualitative aspects of these conclusions are confirmed using standard statistical analysis procedures.

Overall, the GWR and GRA analysis results indicate that, as the travel time increases, the EMS response time also increases, with complex variations as the influences of different numbers and combinations of independent variables are considered. Understanding the effect of these different independent variables on ERT is important, because shortening the ERT minimizes the complications that can ensue related to minor injuries, and major injuries, and reduces the possibility of fatalities. The present research is thus important as it provides enlightenment regarding the statistical dependence of EMS response time, or ERT, on a variety of relevant physical variables, as well as information on the analytical tools which can be utilized for the associated analysis. Such ERT information, and the optimization of associated analysis tools are thus directly relevant for management of care for severely injured surgical patients, optimal trauma patient survivability, and trauma system design.

Future research will consider GWR analysis of the dependence of EMS Response Time (ERT) on a variety of independent variables, as different kernel weighting functions are employed.

\section{Acknowledgements}

The present research did not receive any type of grant support from funding agencies in the public, commercial, or not-for-profit sectors.

\section{Conflicts of Interest}

The authors declare no conflicts of interest regarding the publication of this paper.

\section{References}

Bakke, H. K., Steinvik, T., Eidissen, S. I., Gilbert, M., \& Wisborg T. (2015). Bystander First Aid in Trauma-Prevalence and Quality: A Prospective Observational Study. Acta 
Anaesthesiologica Scandinavica, 59, 1187-1193. https://doi.org/10.1111/aas.12561

Brown, J., Sajankila, N., \& Claridge, J. A. (2017). Prehospital Assessment of Trauma. Surgical Clinics of North America, 97, 961-983. https://doi.org/10.1016/j.suc.2017.06.007

Byrne, J. P., Mann, N. C., Dai, M., Mason, S. A., Karanicolas, P., Rizoli, S., \& Nathens, A. B. (2019). Association between Emergency Medical Service Response Time and Motor Vehicle Crash Mortality in the United States. JAMA Surgery, 154, 286-293. https://doi.org/10.1001/jamasurg.2018.5097

Eftekhari, A., Khorasani-Zavareh, D., \& Nasiriani, K. (2018). The Importance of Designing a Preventable Deaths Instrument for Road Traffic Injuries in Pre-Hospital Phase. Health in Emergencies and Disasters Quarterly, 3, 177-178. https://doi.org/10.32598/hdq.3.4.177

Eftekhari, A., Tafti, A. D., Nasiriani, K., Hajimaghsoudi, M., Fallahzadeh, H., \& Khorasani-Zavareh, D. (2019). Management of Preventable Deaths due to Road Traffic Injuries in Prehospital Phase: A Qualitative Study. Archives of Academic Emergency Medicine, 7, e32.

Evanco, W. M. (1999). The Potential Impact of Rural Mayday Systems on Vehicular Crash Fatalities. Accident Analysis and Prevention, 31, 455-462.

https://doi.org/10.1016/S0001-4575(98)00083-9

Gonzalez, R. P., Cummings, G. R., Phelan, H. A., Mulekar, M. S., \& Rodning, C. B. (2009). Does Increased Emergency Medical Services Prehospital Time Affect Patient Mortality in Rural Motor Vehicle Crashes? A Statewide Analysis. The American Journal of Surgery, 197, 30-34. https://doi.org/10.1016/j.amjsurg.2007.11.018

Gopalakrishnan, S. (2012). A Public Health Perspective of Road Traffic Accidents. Journal of Family Medicine and Primary Care, 1, 144-150. https://doi.org/10.4103/2249-4863.104987

Griffin, R., \& McGwin, G. (2013). Emergency Medical Service Providers' Experiences with Traffic Congestion. The Journal of Emergency Medicine, 44, 398-405. https://doi.org/10.1016/j.jemermed.2012.01.066

He, Z., Qina, X., Renger, R., \& Souvannasacd E. (2019). Using Spatial Regression Methods to Evaluate Rural Emergency Medical Services (EMS). American Journal of Emergency Medicine, 37, 1633-1642. https://doi.org/10.1016/j.ajem.2018.11.029

Insurance Institute for Highway Safety Highway Loss Data Institute (2015). General Statistics. https://www.iihs.org/api/datastoredocument/status-report/pdf/50/1

Kumar, A., Abudayyeh, O., Fredericks, T., Kuk, M., Valente, M., \& Butt, K. (2017). Trend Analyses of Emergency Medical Services for Motor Vehicle Crashes: Michigan Case Study. Transportation Research Record: Journal of the Transportation Research Board, 2635, 55-61. https://doi.org/10.3141\%2F2635-07

Lambert, T. E., \& Meyer, P. B. (2006). Ex-Urban Sprawl as a Factor in Traffic Fatalities and EMS Response Times in the Southeastern United States. Journal of Economic Is sues, 40, 941-953. https://doi.org/10.1080/00213624.2006.11506968

Ma, L., Zhang, H., Yan, X., Wang, J., Song, Z., \& Xiong, H. (2019). Smooth Associations between the Emergency Medical Services Response Time and the Risk of Death in Road Traffic Crashes. Journal of Transport and Health, 12, 379-391. https://doi.org/10.1016/j.jth.2018.08.011

Nakaya, T. (2014). GWR4 User Manual. GWR 4 Development Team.

National Academies of Sciences, Engineering, and Medicine. Medicine. (2016). A National Trauma Care System: Integrating Military and Civilian Trauma Systems to Achieve Zero 
Preventable Deaths After Injury. The National Academies Press.

National Highway Traffic Safety Administration (2017). FARS Annual Crash Statistics. https://crashstats.nhtsa.dot.gov/Api/Public/ViewPublication/812794

NCHRP (National Cooperative Highway Research Program) (2013). Synthesis of Highway Practice 451: Emergency Medical Services Response to Motor Vehicle Crashes in Rural Areas. Transportation Research Board, National Research Council. http://onlinepubs.trb.org/onlinepubs/nchrp/nchrp syn 451.pdf

Peden, M., Scurfield, R., Sleet, D., Mohan, D., Hyder, A. A., \& Jarawan, E. (2004). World Report on Road Traffic Injury Prevention. World Health Organization.

Pons, P. T., Haukoos, J. S., Bludworth, W., Cribley, T., Pons, K. A., \& Markovchick, V. J. (2005). Paramedic Response Time: Does It Affect Patient Survival? Academic Emergency Medicine, 12, 594-600.

Trowbridge, M. J., Gurka, R. E., \& O'Connor, R. E. (2009). Urban Sprawl and Delayed Ambulance Arrival in the U.S. American Journal of Preventive Medicine, 37, 428-432. https://doi.org/10.1016/j.amepre.2009.06.016

World Health Organization (2013). Global Status Report on Road Safety 2013: Supporting a Decade of Action. World Health Organization.

World Health Organization (2015). Ten Facts on Global Road Safety. World Health Organization. https://www.who.int/features/factfiles/roadsafety/en/ 